\title{
Endoscopic Management of Postlaparoscopic Sleeve Gastrectomy Leaks: A Single-Center Experience
}

\author{
Nitin Jagtap ${ }^{1, \odot}$ H.S. Yashavanth ${ }^{1}$ Rakesh Kalapala ${ }^{1}$ Abhishek Katakwar ${ }^{2}$ Mohan Ramchandani ${ }^{1}$ \\ Vaibhav Ajmere $^{1} \quad$ Manu Tandan $^{1}$ Santosh Darishetty ${ }^{3} \quad$ G. Venkat Rao ${ }^{2} \quad$ D. Nageshwar Reddy ${ }^{1}$
}

${ }^{1}$ Department of Medical Gastroenterology, Asian Institute of Gastroenterology, Hyderabad, Telangana, India

${ }^{2}$ Department of Surgical Gastroenterology, Asian Institute of

Gastroenterology, Hyderabad, Telangana, India

${ }^{3}$ Department of Anaesthesiology, Asian Institute of

Gastroenterology, Hyderabad, Telangana, India

J Digest Endosc 2020;11:134-137
Address for correspondence Nitin Jagtap, MD, DNB, Department of Medical Gastroenterology, Asian Institute of Gastroenterology, 6-3-661, Somajiguda, Hyderabad 500082, Telangana, India (e-mail: docnits13@gmail.com).

\begin{abstract}
Keywords

- obesity

- laparoscopic sleeve gastrectomy

- leaks

- endoscopy

- mega SEMS

Introduction Laparoscopic sleeve gastrectomy (LSG) is a commonly performed bariatric surgery. Sleeve leak is the most important complication, with an incidence of 1.9 to $2.4 \%$. Various endoscopic approaches to LSG have been used, including selfexpanding metal stents (SEMSs), glue injection, and clipping along with percutaneous drainage. This study was aimed to study the role of endotherapy in the management of post-LSG leaks.

Methods This study included patients referred for endotherapy for post-LSG leak between January 2016 and December 2018. We maintained data prospectively, which included the location and type of leak, type of endotherapy, adverse events, and time for leak closure. Primary endotherapy included mega SEMS placement; if it failed, then secondary endoscopic therapy was performed.

Results Seven patients (four females, with a mean age of 45.2 years) with a preoperative body mass index of mean $38.5 \mathrm{~kg} / \mathrm{m}^{2}$ underwent endotherapy for post-LSG leaks. Two were acute, four were early, and the remaining one was late leak. Five were located proximally near gastroesophageal junction and two at the midsleeve level. In four $(57.1 \%)$ patients, the leak was resolved by primary therapy. Three patients underwent secondary therapy that included overlapping SEMS placement (in one patient), SEMS replacement (in one patient), and short plastic biliary stent placement with Argon plasma coagulation (APC) to create a raw surface and induce granulation tissue. The median duration for leak closure was 12 weeks (range: $8-24$ weeks). One patient who had partial distal migration underwent overlapping SEMS placement. Three patients had nonbleeding ulcers at the distal end of SEMS at removal.

Conclusion Endotherapy is effective and safe for the management of post-LSG leaks. Additional endotherapy can be used if primary therapy is not successful for resolution of the leak.
\end{abstract}

\section{Introduction}

The epidemic of obesity in India causes nearly 5.8 million deaths per year., ${ }^{1,2}$ Currently, obesity and its associated comorbidities such as diabetes mellitus are major cause of health concern in India. ${ }^{3}$ Metabolic and bariatric therapies such as endoscopic and surgical interventions are essential to treat this subset of population. ${ }^{2,4}$ Among the bariatric and metabolic surgeries, laparoscopic sleeve gastrectomy (LSG) is one the most commonly performed procedure. However, 
LSG is associated with several adverse events such as bleeding, leaks, and stenosis. Post-LSG leak is associated with significant morbidity and mortality, with a reported incidence of 1.9 to $2.4 \%{ }^{5,6}$ Various endoscopic therapies have been described as minimally invasive and effective alternatives for the management of post-LSG leaks, which include selfexpanding metal stents (SEMSs), glue injection, and clips along with percutaneous drainage of perigastric collection..$^{7-9}$

In this study, we aim to evaluate the role of endoscopic therapies for post-LSG leaks at a single tertiary care referral center.

\section{Methods}

Institutional Review Board approval was obtained for the study. This study included patients referred for endotherapy for post-LSG leaks between January 2016 and June 2018. Sleeve leak was diagnosed by clinical symptoms of pain and signs of sepsis, if present, along with evidence of leak on oral contrast study, cross-sectional imaging, and upper gastrointestinal endoscopy. We maintained data prospectively, which included location and type of leak, type of endotherapy, adverse events, and time required for leak closure. Post-LSG leaks ( - Fig. 1) were classified as acute (first postoperative week 1 ), early (postoperative weeks $1-6$ ), late (postoperative weeks $6-12$ ), and chronic (postoperative week 12 or more). ${ }^{10}$ Primary endotherapy includes mega SEMS (Taewoong Niti-S Megastent, Cook Medical, Bloomington, Indiana, United States) placement (-Fig. 2). Patients were advised to start liquid diet from the second day of the procedure, and, if tolerated, to start soft diet from the third to fifth day. Afterward, patients were advised for regular diet as advised by a trained nutritionist. Patients were advised for follow-up

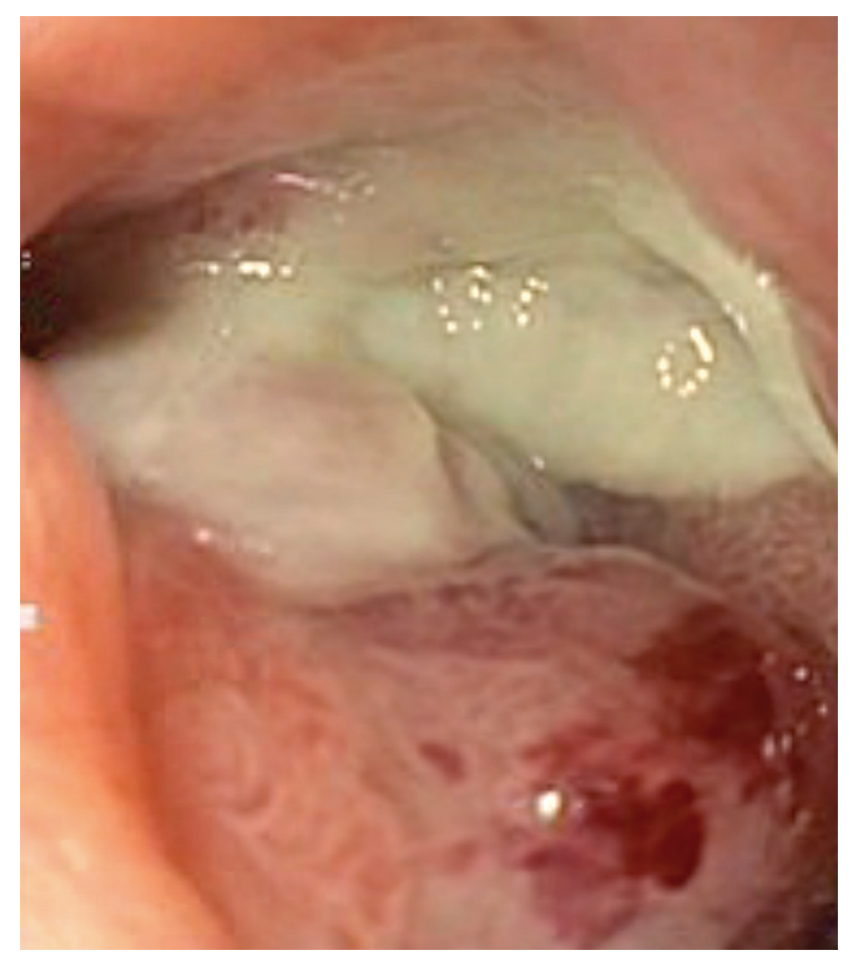

Fig. 1 Endoscopic view of postlaparoscopic sleeve gastrectomy leak.

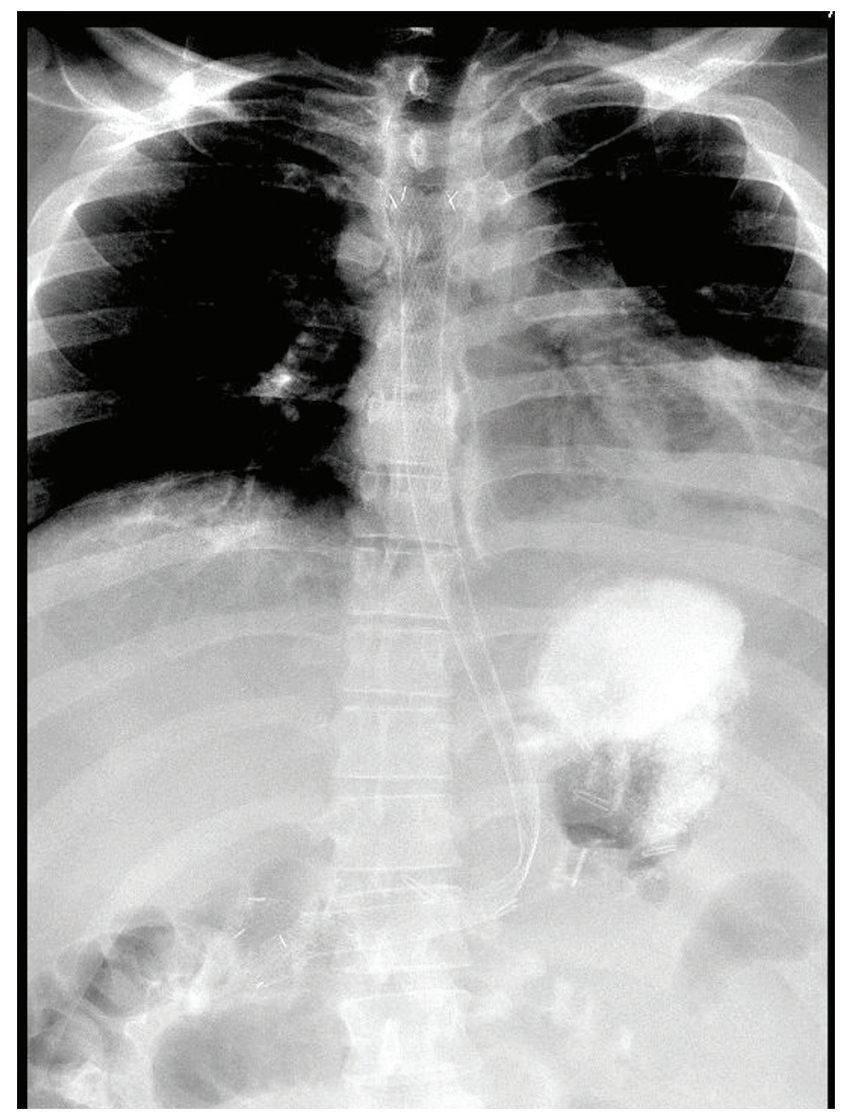

Fig. 2 Fluoroscopy showing mega self-expanding metal stent.

after 8 to 12 weeks for the removal of SEMS and the assessment of leak closure or early if clinically indicated. If the leak closure failed, then a secondary endoscopic therapy was performed, which includes either SEMS replacement or overlapping SEMS placement or other endotherapies such as APC to create a raw surface to induce tissue reaction, which will promote leak closure and short plastic biliary stent placement to facilitate internal drainage of perigastric collection, and so on. Overlapping SEMS was placed in case of partial distal migration, whereas SEMS was replaced if significant mucosal defect is present at removal of the initial SEMS. Other secondary endotherapies were considered for small leaks. Leak closure was documented by oral contrast study and endoscopy. Perigastric collections were drained by ultrasound or computed tomography (CT) guided percutaneous drain placement whenever clinically indicated.

\section{Results}

Between January 2016 and December 2018, seven patients were referred for endoscopic management of leaks following LSG performed at other institutions. Of the seven patients, three $(43 \%)$ were males and $4(57 \%)$ were females, with a mean age of 45.2 years (range: $31-55$ years). The mean preoperative body mass index was $38.5 \mathrm{~kg} / \mathrm{m}^{2}$ (range: $32.3-54.7 \mathrm{~kg} / \mathrm{m}^{2}$ ). Two leaks were acute, four were early, and one was late. Majority (five) leaks were located proximally near the gastroesophageal junction and two were midsleeve leaks. No patients had multiple staple line leaks. 
Six patients underwent ultrasound or CT-guided percutaneous drainage of perigastric collection before endoscopic therapy, and one patient had small collection, which was managed with ultrasound-guided single-time aspiration. All seven patients had leak resolution either by primary therapy (four patients [57.1\%]) or secondary therapy (one with overlapping SEMS placement, one with SEMS replacement, and one with APC and short plastic stent placement) at a median of 12 weeks (range: $8-24$ weeks). We placed SEMS as primary therapy for 8 to 12 weeks. Four patients achieved leak healing with a single SEMS placement. SEMS was removed before 12 weeks in these patients. One patient required overlapping SEMS placement at the sixth week for partial distal migration; both stents were removed at 16 weeks. Another patient underwent SEMS replacement at 10th week for persistent leak after the first SEMS removal with complete healing and stent extraction at 19th week. One patient underwent short plastic biliary stent placement for internal drainage of small perigastric collection and APC to create a raw surface and induce granulation tissue. Minor adverse events (in two patients) such as pain, nausea, and vomiting were resolved with conservative treatment. There were nonbleeding ulcers at the distal end of the stent due to wide distal flare in three patients.

\section{Discussion}

LSG is one of the most commonly performed bariatric surgery. Post-LSG leak is an important complication, with an overall incidence of 1.9 to $2.4 \%$. Various therapeutic strategies have been described for its management based on the clinical presentation. A prompt laparoscopic drainage with an omental patch or primary closure of defect is ideal for patients with clinical instability. However, a stable patient with leak can be managed with endoscopic intervention along with percutaneous drainage of perigastric collection.

Our study has shown an overall success of $100 \%$ at a median of 12 weeks (range: 8-24 weeks). Overall, $57.1 \%$ of patients (4/7) achieved leak closure with primary endotherapy by SEMS placement and 28.6\% (2/7) achieved leak closure with additional SEMS placement. One patient underwent APC and short plastic stent placement after failure of SEMS. There were no serious adverse events related to endotherapy except for one partial distal migration, and four patients had nonbleeding ulcers at the distal end of the stent.

Endotherapy for post LSG leaks is a minimally invasive therapeutic option with early ambulation, short hospitalization and allows initiation of enteral feeding. ${ }^{11,12}$ The development of post LSG leaks is attributed to gastric wall ischemia, increased intraluminal pressure due to low compliance of gastric sleeve, and stenosis of the sleeve. ${ }^{10,13}$ After placement of SEMS, intraluminal pressure will be reduced and enteral nutrition can be allowed as stent will bypass the leak and aids in healing.

We used Taewoong Niti-S Megastent $(24 \mathrm{~mm}$ wide and $230 \mathrm{~mm}$ long with two 32-mm-wide flared ends) as primary endotherapy. This specially designed stent has a low risk of migration and covers the entire length of the gastric sleeve to provide effective drainage, which is major determinant for leak healing. ${ }^{11,14}$ SEMS-related adverse events include intolerance, hemorrhage, migration, reflux, and stent-related stricture formation. In this study, three patients developed stent-related ulcers and one patient had partial migration. There was technical difficulty in retrieval of partially migrated SEMS, and therefore the patient underwent overlapping SEMS placement to facilitate retrieval using SEMS in the SEMS technique. ${ }^{15,16}$ SEMS replacement was performed in one patient as there was significant mucosal defect after retrieval of the initially placed SEMS.

A single-center study of 21 patients from New Zealand has shown $95 \%$ post-LSG leak closure at a mean duration of 10.7 weeks of endoscopic therapy, with a $19 \%$ migration rate. ${ }^{9}$ A modified SEMS Taewoong Niti- S Beta II $(24 \times 200 \mathrm{~mm}$ double antimigratory cuffed stent) with uncovered proximal flare $(10 \mathrm{~mm})$ has shown reduced inhospital stay and time required for leak closure. ${ }^{9}$ Another study of 81 patients has shown $82 \%$ leak closure with stent migration in $18 \%$ patients with stent-related mortality due to bleeding. ${ }^{17}$ Hamed et al recently reported $73.3 \%$ clinical success with a $17.8 \%$ migration rate. ${ }^{12}$ This study has shown $85.7 \%$ (six of seven patients: four patients with single SEMS, two with two SEMS) success rate with SEMS with a $14.3 \%$ migration rate.

The placement of short plastic stent endoscopically in perigastric collection can provide internal drainage. This therapeutic option has a $95 \%$ success rate, with a mean of 55.5 days, till leak closure. ${ }^{18}$ However, this approach should be reserved for leaks smaller than $10 \mathrm{~mm}$. We used this approach along with APC as secondary therapy in one patient in whom small leak was persistent after SEMS removal. We consider endoscopic mega SEMS as the primary option for large leaks and other supplementary therapies such as internal drainage with plastic stents, metal clips, OTSC clips (OTSC, Ovesco AG, Tuebingen, Germany), and APC if primary therapy fails. Endoscopic suturing using Apollo Overstitch Suturing System (Apollo Endosurgery Inc., Austin, Texas, United States) is an attractive approach for leak closure if it is technically feasible. ${ }^{19,20}$ The use of supplementary endotherapy should be determined by endoscopic appearance of leak, local expertise, and patient preference.

There are a few limitations of this study apart from being retrospective. This series does not include all consecutive patients of post-LSG leaks but only who were referred for endotherapy for post-LSG leaks from other institutes.

In conclusion, post-LSG leaks can be managed by endotherapy with significant success rates and acceptable morbidity. The improvement in stent designs along with supplementary use of other endoscopic therapeutic modalities can further improve clinical success in such patients.

\section{Authors Contribution}

Nitin Jagtap contributed to the designed study, Yashavanth HS and Vaibhav Ajmere contributed to the data collection, Nitin Jagtap and Abhishek Katakwar contributed to critical analysis and data interpretation, Nitin Jagtap and Rakesh Kalapala contributed to preparing the draft of the article, Abhishek Katakwar and Mohan Ramchandani 
provided intellectual inputs, Rakesh Kalapala and Manu Tandan contributed to the critical revision of the article, and G. Venkat Rao and D. Nageshwar Reddy contributed to the final approval of the manuscript. All authors had unlimited access to data and manuscript.

\section{Ethics Statement}

The authors declare that the study was performed in accordance with the Helsinki Declaration of 1975, as revised in 2000 and 2008, concerning human and animal rights. The protocol was approved by the Institutional Ethics Committee, and informed consent was obtained from the patients. The authors are responsible for the findings and the content of the paper.

\section{Conflict of Interest}

The authors declare no conflict of interest.

\section{References}

1 Shrivastava U, Misra A, Mohan V, Unnikrishnan R, Bachani D. Obesity, diabetes and cardiovascular diseases in India: public health challenges. Curr Diabetes Rev 2017;13(1):65-80

2 Jagtap N, Kalapala R, Katakwar A, Darishetty S, Reddy D. Endoscopic sleeve gastroplasty for obesity - first Indian experience: case series and review of literature. J Dig Endosc 2018;9:165

3 Anjana RM, Deepa M, Pradeepa R, et al; ICMR-INDIAB Collaborative Study Group. Prevalence of diabetes and prediabetes in 15 states of India: results from the ICMR-INDIAB population-based cross-sectional study. Lancet Diabetes Endocrinol 2017;5(8):585-596

4 Ohta M, Seki Y, Wong SK, et al. Bariatric/metabolic surgery in the Asia-Pacific Region: APMBSS 2018 survey. Obes Surg 2019;29(2):534-541

5 Benedix F, Poranzke O, Adolf D, et al; Obesity Surgery Working Group Competence Network Obesity. Staple line leak after primary sleeve gastrectomy-risk factors and mid-term results: do patients still benefit from the weight loss procedure? Obes Surg 2017;27(7):1780-1788

6 Aurora AR, Khaitan L, Saber AA. Sleeve gastrectomy and the risk of leak: a systematic analysis of 4,888 patients. Surg Endosc 2012;26(6):1509-1515

7 Puli SR, Spofford IS, Thompson CC. Use of self-expandable stents in the treatment of bariatric surgery leaks: a systematic review and meta-analysis. Gastrointest Endosc 2012;75(2): 287-293
8 Corona M, Zini C, Allegritti M, et al. Minimally invasive treatment of gastric leak after sleeve gastrectomy. Radiol Med (Torino) 2013;118(6):962-970

9 Southwell T, Lim TH, Ogra R. Endoscopic therapy for treatment of staple line leaks post-laparoscopic sleeve gastrectomy (LSG): experience from a large bariatric surgery centre in New Zealand. Obes Surg 2016;26(6):1155-1162

10 Rosenthal RJ, Diaz AA, Arvidsson D, et al; International Sleeve Gastrectomy Expert Panel. International Sleeve Gastrectomy Expert Panel Consensus Statement: best practice guidelines based on experience of $>12,000$ cases. Surg Obes Relat Dis 2012;8(1):8-19

11 Tsai YN, Wang HP, Huang CK, Chang PC, Lin IC, Tai CM. Endoluminal stenting for the management of leak following sleeve gastrectomy and loop duodenojejunal bypass with sleeve gastrectomy. Kaohsiung J Med Sci 2018;34(1):43-48

12 Hamed H, Said M, Elghadban H, Elgeidie A. Outcome and adverse events of endoscopic bariatric stents for management of leakage after bariatric surgery. Obes Surg 2020;30(3):982-991

13 Courcoulas AP, Yanovski SZ, Bonds D, et al. Long-term outcomes of bariatric surgery: a National Institutes of Health symposium. JAMA Surg 2014;149(12):1323-1329

14 Basha J, Appasani S, Sinha SK, et al. Mega stents: a new option for management of leaks following laparoscopic sleeve gastrectomy. Endoscopy 2014;46(Suppl 1 UCTN) :E49-E50

15 Tringali A, Blero D, Boškoski I, et al. Difficult removal of fully covered self expandable metal stents (SEMS) for benign biliary strictures: the "SEMS in SEMS" technique. Dig Liver Dis 2014;46(6):568-571

16 Chandrasekar TS, Kalamegam RY, Janakan GB, Suriyanarayanan S, Menta PS, Chandrasekar VT. Double-step invagination technique-a novel approach to retrieve embedded esophageal self-expanding metal stent. Indian J Gastroenterol 2017;36(5):424-428

17 Shehab H, Abdallah E, Gawdat K, Elattar I. Large bariatric-specific stents and over-the-scope clips in the management of post-bariatric surgery leaks. Obes Surg 2018;28(1):15-24

18 Donatelli G, Ferretti S, Vergeau BM, et al. Endoscopic internal drainage with enteral nutrition (EDEN) for treatment of leaks following sleeve gastrectomy. Obes Surg 2014;24(8):1400-1407

19 Sharaiha RZ, Kumta NA, DeFilippis EM, et al. A large multicenter experience with endoscopic suturing for management of gastrointestinal defects and stent anchorage in 122 patients: a retrospective review. J Clin Gastroenterol 2016;50(5):388-392

20 Jagtap N, Kalapala R, Rao GV, Reddy DN. Endoscopic suturing for refractory bilious reflux after gastroduodenostomy and gastrojejunostomy. Endoscopy 2019;(e-pub ahead of print). doi:10.1055/a-1063-6251 